VOL 3 (2019) NO 1

e-ISSN : 2549-9904

ISSN : 2549-9610

\title{
Metadata Extraction Analysis: A Review of Video Data in Effect to Social Media Compression
}

\author{
Dawn Iris Calibo ${ }^{\#}$, Jasmin D. Niguidula* \\ \# Siquijor State College, Larena, Siquijor, 6226, Philippines \\ ${ }^{*}$ Technological Institute of the Philippines-Manila, Quiapo, Manila, 1000, Philippines \\ E-mail: dawniris_19@yahoo.com, jasniguidula@yahoo.com
}

\begin{abstract}
In the 21st century, the unending improvement of the web, online networking has made approaches to take advantage to streamline the speed of its webpage. Through metatadta extraction, two of the most well-known social networking sites were subjected to experimentation to see the impacts of video compression using standard parameters when uploaded to the said sites. The assessment demonstrates that both sites display keys similarities and differences. It is further explained in the research the different structures amongst the social network that create the outcome.
\end{abstract}

Keywords - Video Compression, Metadata Extraction, Video Analysis, Social Media

\section{INTRODUCTION}

Social media networking is computer intervened advancements that permit the creation and sharing of data, thoughts, profession interests, and different types of expression by means of virtual groups and systems. Their regular elements incorporate being intelligent, having user created content and producing information through every single online communication. Online networking users benefit on profiles for the site or application that are composed and kept by the web-based social networking media [1].

Facebook.com is an online web-based social networking and long range interpersonal communication benefit where users can include different users as "companions", trade messages, post notices and digital photographs, share advanced recordings and connections, utilize different applications and get updates on others [2]. Another social network is Youtube.com, a video-sharing site that permits users to make and update individual accounts. The website additionally gives a stage to uploading and sharing video clips, Network program cuts, music recordings, short and narrative movies, motion picture trailers and other substance, like, blogging, short unique recordings, and e-learning videos that are transferred by people, media companies, and different associations as a component of the YouTube organization program.

As cited in the work of Seymour [3] typically, the expansion in web users who are watching video via social media whether in Facebook or Youtube and others affects the video itself. According to study, social video is significantly affecting on the web video in general. The article states that video length has contracted and has even required the inclinations of social media gatherings of people who take the opportunity to segment videos. However, Peter Hawley an expert in film and broadcast views that this is something to look forward. He further explains that the most impactful recordings nowadays are short, direct to the point, and should be easily shareable.

In general, the normal number of recordings and pictures transferred to a website page continues to progress, while the expansion in web speed has not deal with the development of the normal page measure. The web is the increment in substance without a comparing increment in the speed of web associations has prompted to the improvement data compression [4].

Video quality is a characteristic of a video that went through a video transmission system which is measured to get video compression. Video processing frameworks may present some measure of distortion in the video signal, which contrarily impacts the user's idea of a system. For service providers and system administrators, the affirmation of video quality is a vital task [5].

This paper aims to evaluate the factors that affect video quality and its structure, especially as they relate to social media compression. This leads to the evaluation of the following contributing factors to video quality in effect of social media compressions such as video duration, video 
width, video height, video average bitrates, video frame rate, audio duration audio bitrates, audio channels number, and audio sampling rate.

\section{LITERATURE REVIEW}

Compression in information theory is the system utilized as a part of compressed records, Portable Network Graphics documents (compressed pictures), and MPEG Audio Layer 3 data (compacted sound) to lessen the span of an informational index (the quantity of bits) to its smallest size while limiting the measure of data lost simultaneously. Algorithmic compression recognizes examples and develops a code that interprets each as often as possible showing up series of bits into a much shorter string, making it pointless to reproduce the whole original string in each of its appearances. Compression along these lines wipes out excess. Its objective is productivity, finding the minimum string of bits (called the algorithmic information content) that is important to transmit data [6].

Poynton, [7] further discussed that because data compression has the objective of lessening the quantity of bit required to store or pass on content video quality is gotten by neglecting measurable properties of the information. The lessening comes to the detriment of the computational drive to compress and decompress. He added that the direct storing or transmitting of digital video requires high data capacity evaluated 20 megabytes per second for standard definition (SD) or about 120 megabytes per second for high definition (HD). This administrates the rule that relates storage capacity and data rate where: Eight (8), 2000-feet (ft) reels of motion picture print film can hold $1131 / 3$ minute movie; there are eight (8) bits in a byte and 60 seconds in a minute; and 60/8. $1131 / 3$ is 1000 . So one (1) megabyte per second equals one gigabyte per movie is the computation whether the video is compressed or not. In the same manner, one gigabyte per second equals one terabyte per movie. This is represented by the following equations:

\section{$\mathrm{Mb} / \mathrm{s}=\mathrm{GB} / \mathrm{movie}$ and $\mathrm{Gb} / \mathrm{s}=\mathrm{TB} / \mathrm{movie}$}

Whenever videos/movies are transferred in social media platforms this can be downloaded and process for compression analysis. This is done through metadata extraction where video data are extricated from container or wrapper format.

Metadata is a data that gives information to another set of data [8]. Such metadata can be classified into three namely; descriptive, structural and administrative. Descriptive depicts a system which deals with the disclosure and recognizable proof such as title, creator, abstract and catchphrases. Structural, on the other hand, deals with the metadata containers and explains how composite things are being assembled. The last type of metadata is administrative which give information in handling resources [9].

Video editing software in recent development can particularly penetrate metadata. Metadata is considered important in video content since it lead on to conveniently search for details. Identification of metadata through different sources is important. An example is the operational gathered metadata where information about the substance created, like, software and hardware specification, programming date, and area. Another is the human-authored metadata which aims to promote video visibility and performance beneficial to developers [10].

In the analysis of metadata extraction metafile format must be identified. A container or wrapper format is a metafile format whose particular portrays how distinctive components of data and metadata exist together in a computer file. The container doesn't represent how data or metadata is encoded and so a program that identifies and opens a container is used to decode the contained data. An example of such is the Elecard Format Analyzer.

In the work of Poynton [11], he discussed that video compression, the Broadcast Wave Format (BWF) file which is known for broadcast audio encompasses a parameter called nSamplesPerSec giving the sample rate. The parameter is contained in a BWF Metadata Chunk. As introduced by Abekas in 1980s, the yuv file format is used to store uncompressed video. Given when 8 -bit $\mathrm{Y}^{\prime} \mathrm{C}_{\mathrm{B}} \mathrm{C}_{\mathrm{R}}$ is for 4:2:2 interweaved video raster order, the file format definition may be as follows: First, store successive image rows, given that each row is a sequence of 4-byte elements $\left[\mathrm{C}_{\mathrm{BO}}, \mathrm{Y}_{\mathrm{O}}{ }^{\prime}, \mathrm{C}_{\mathrm{RO}}, \mathrm{Y}_{1}{ }^{\prime}\right]$ and where subscript $\mathrm{O}$ represents an even-numbered luma sample location ad subscript 1 represents odd. It is noted that there is no header in a yuv file and there is a no provision of storing the count of frames, image row, or columns. This format was then introduced to the market as $720 \times 480$ video. Later on, it is applied to $720 \times 576$ and became potentially applicable to $720 \times 486$, or $704 \times 480$. The codec research community then utilized 1280x720p and 1920x1080i. To continue, examine a .yuv records compelled to be $720 \times 480$ or $720 \times 576$. Mostly the configuration can be measured by extrication of the record's bytecount by 1440 and dividing it by 480 and 576 thus to see which quotient is an integer. However, this approach has uncertainties. Thus, reliable file interpretation is achieved only from the agreement between the sender and receiver such that of social media platforms and the users. This is expressed more properly in terms and conditions between writer and reader.

To sum it up, what contains metadata relies on upon the client considers to be the system. The bigger, more comprehensive, and broader the system becomes and the less it depends on setting than the more metadata transforms into data.

\section{METHODOLOGY}

\section{A. Video Compression Tool}

This research utilizes a software tool known as Elecard Video Format Analyzer. Its features include extraction and examination of metadata enclosed in media records. This particularly scrutinizes data as far as in terms of length, size, bitrates and more. This apparatus executes the accompanying components that enable video and audio compression evaluation. It likewise gives hexadecimal review form for document and its chunks comparing to the predetermined portions. In addition, text representation of a document is shown in the preview pane. It permits simple interference of the parsing procedure at any given minute in the handling of information. 


\section{B. Formulation}

The experimental method was used in this research as it manipulates one variable, and controls the rest of the variables. In this case, two widely used social media website was the subject of the experiment namely facebook.com and youtube.com.

For facebook.com, five recordings with a similar video length of 4:45 minutes or 285 seconds were utilized. While for youtube.com, an original video made using Wondershare Filmora entitled 21 Traipse of a Man which was used in Youtube.com last October 24, 2016. The original video has been subjected to testing through uploading and downloading strategy for nine (9) trials to get the data required by the review. The recordings are then transferred to Elecard Video Format Analyzer for examination utilizing the accompanying parameters:

TABLE I

PARAMETERS FOR IDENTIFYING VIDEO AND AUDIO COMPRESSION FINDINGS

\begin{tabular}{|c|c|}
\hline $\begin{array}{c}\text { Parameters for Video } \\
\text { Compression Analysis }\end{array}$ & $\begin{array}{c}\text { Parameters for Audio } \\
\text { Compression Analysis }\end{array}$ \\
\hline Duration & Duration \\
\hline Width & Bitrate \\
\hline Height & Channels number \\
\hline Average bitrate & Sampling rate \\
\hline Frame rate & \\
\hline
\end{tabular}

\section{RESULTS AND DISCUSSION}

This part of the study shows the metadata extraction results based on the different parameters from the video compression tool.

In accordance with facebook.com standards, users are permitted to share videos with immense bitrates even up to 1 Gigabyte in size. In such case, the maximum file size that is 1.75 gigabyte and the time length is 45 minutes. In the case a user go past a 1-gigabyte file size, then the user should restrain a 1080 high definition (HD) upload file to a maximum bitrate of $8 \mathrm{Mbps}$, and a 720 high definition (HD) upload file must be restricted to a maximum bitrate of 4 Mbps.

Since the research used a video that didn't surpass the maximum length, the videos have been successfully uploaded. However, during the metadata extraction, it was found out that it is notable that original videos present a remarkable change after it has been uploaded to the site. This is because the ideal Facebook.com video dimension is 720 p whose frame size of 1280 px wide by 720px high and on the off chance that you upload a video that contains higher resolution then Facebook.com will scale back the video. On the off chance that you let Facebook.com resize the video frame, the user may see that the quality in the process will be distorted [12].

In figure 1 , the different original videos transferred to facebook.com accomplish even outcomes after it has been compressed is shown. To be specific it is noted that all the original video duration didn't change after it has been compressed. For the video width and height, similarly, all the original video using $1280 \times 720$ are compressed to $400 \times 224$. For the average bitrate, the outcome demonstrates that $11 \%$ of the evaluated bitrate is detracted from the original video. It further shows that for the frame rate both the original and compressed videos in data 2, 3, 4 and 5 remains the same adjacent to from the first original video where it gets to be distinctly 0.01 higher after it has been compressed.

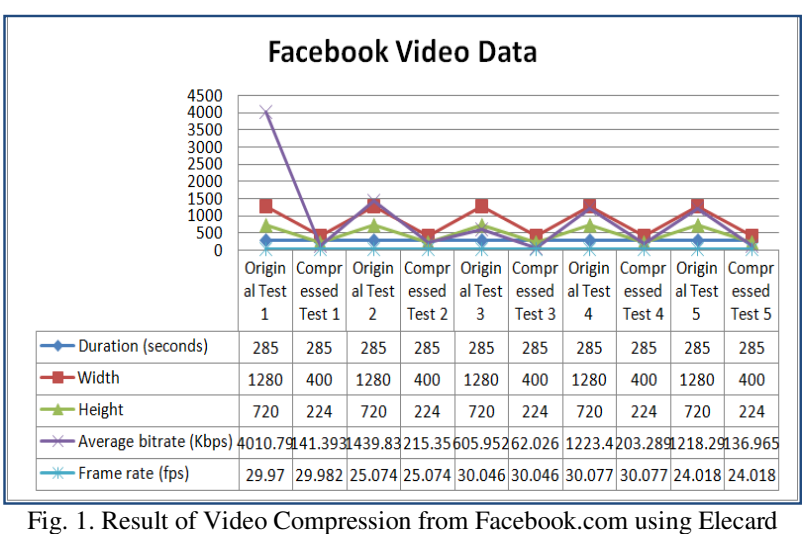

Video Format Analyzer

Figure 2 illustrates the impacts of video compression with regards to the audio data of the original video when transferred to facebook.com. The outcome demonstrates that audio duration and channel of all five of the original and compressed data was not altered. For the bitrate the result reveals that $16 \%$ of the evaluated bitrate has been detracted from the original audio. It further demonstrates that for the sampling rates both the original and compressed audios in data 2, 3, 4 and 5 remains the same comparable. However, the first original audio with the rate of 0.029 gets to be noticeably 44.071 higher after it has been compressed. This result compliments the facebook.com compression effects on video from the previous discussion.

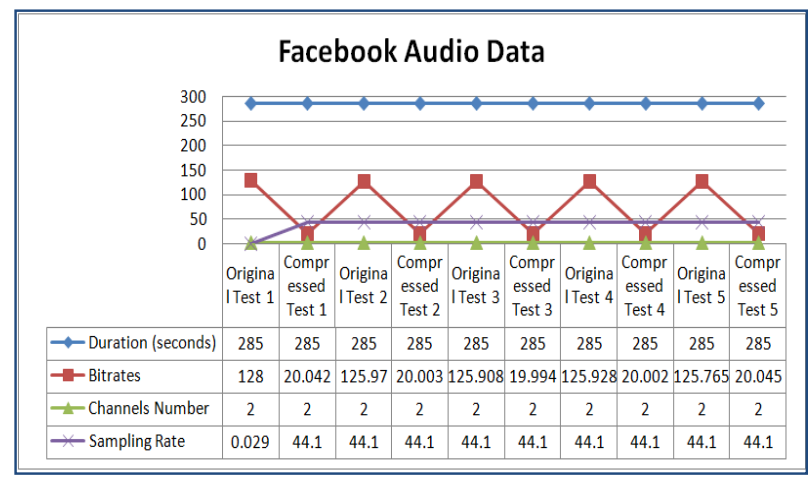

Fig. 2. Result of Audio Compression from Facebook.com using Elecard Video Format Analyzer

In the case of youtube.com, it has been observed that the compression rates of its video and audio data amid the experimentation is not exceptionally altered The minimal result of compression is due to the fact that, Youtube, as a video site, has keep track with the demand of video writer and reader by continually upgrading the site for user's satisfaction. In the beginning Youtube.com offered videos at only one quality level [13].

Its technology later goes through series of upgrades as presented in the table below: 
TABLE III

DEVELOPMENT OF YOUTUBE

\begin{tabular}{|c|c|}
\hline $\begin{array}{l}\text { Progress } \\
\text { Date }\end{array}$ & Format and Quality \\
\hline Original & $\begin{array}{l}\text {-resolution display } 320 \times 240 \text { pixels } \\
\text { - mono MP3 audio }\end{array}$ \\
\hline June 2007 & - 3GP format on mobile phones \\
\hline March 2008 & - resolution $480 \times 360$ pixels \\
\hline $\begin{array}{l}\text { November } \\
2008\end{array}$ & $\begin{array}{l}\text { - 720p high definition (HD) support } \\
\text { - 4:3 aspect ratio to a widescreen 16:9 } \\
\text {-used H.264/MPEG-4 AVC as default for } \\
\text { video compression format }\end{array}$ \\
\hline $\begin{array}{l}\text { November } \\
2009\end{array}$ & -1080p high definition (HD) support \\
\hline July 2010 & $\begin{array}{l}\text { - 4k resolution support } \\
\text { - resolution } 4096 \times 3072 \text { pixels [16] }\end{array}$ \\
\hline June 2014 & - videos playing at 60 frames per second \\
\hline June 2015 & $\begin{array}{l}\text { - 8k resolution support } \\
-7680 \times 4320 \text { pixels [17] }\end{array}$ \\
\hline $\begin{array}{l}\text { November } \\
2016\end{array}$ & $\begin{array}{l}\text { - high dynamic range (HDR) video encoded } \\
\text { with highbred log gamma (HDR) or } \\
\text { perceptual quantizer (PQ) [18] } \\
\text {-video is encoded with Rec. } 2020 \text { color } \\
\text { space }\end{array}$ \\
\hline
\end{tabular}

Table 2 shows the progress of youtube's video standardization to enhance video quality and eradicate the glaring effect of compression to the videos uploaded and downloaded on the site.

Figure 3 , this illustrates the result after the carrying out tests of subjecting an original video through uploading and downloading strategy for various nine (9) trials to get the compression rate once it is uploaded to youtube.com. The result shows that the video duration, width, height and total frames did not change after compression. It additionally demonstrates that for the frame rate shape the compressed videos in test $2,3,4,5,6,7,8$ and 9 remains as before contiguous from the original video where it gets to be evidently - $0.016 \mathrm{fps}$ abase after it has been compressed. For the average bitrate it shows that surprising change is notable in the original video from $4010.797 \mathrm{Kbps}$ was a distinction of $2653.525 \mathrm{Kbps}$ after it was transferred to youtube.com. From that point forward the rest of the 8 trials diminishes its depreciation around $18.537 \mathrm{Kbps}$ in each trial.

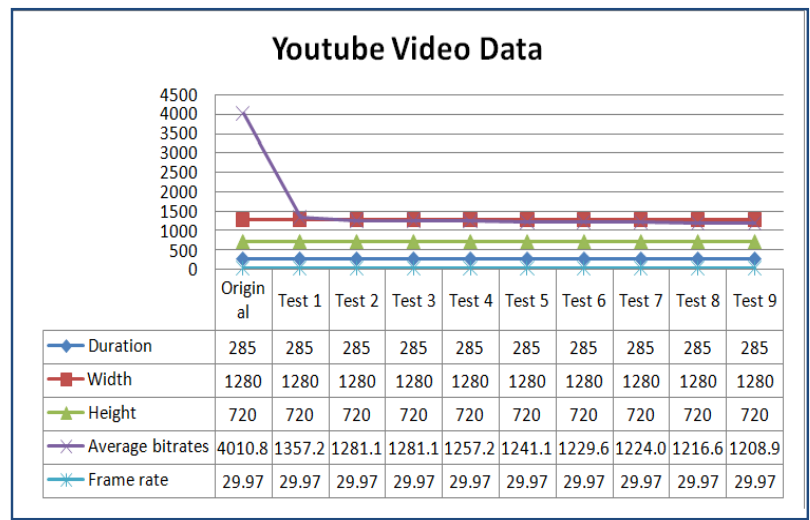

Fig 3. Result of Video Compression from Youtube.com using Elecard Video Format Analyzer
Figure 4 depicts the result of video compression with regards to the audio data of the original video when uploaded to youtube.com. The outcome demonstrates that the audio duration and audio channel of the original and compressed video have not changed. In any case, it demonstrates that the bitrate of the first video has adjusted from $128 \mathrm{Kbps}$ to $192.111 \mathrm{Kbps}$ and continues as before to the rest of the trials. In a similar hand, the sampling rate of the original audio is $0.029 \mathrm{kHz}$ is adjusted to $44.1 \mathrm{kHz}$ on the rest of the trials.

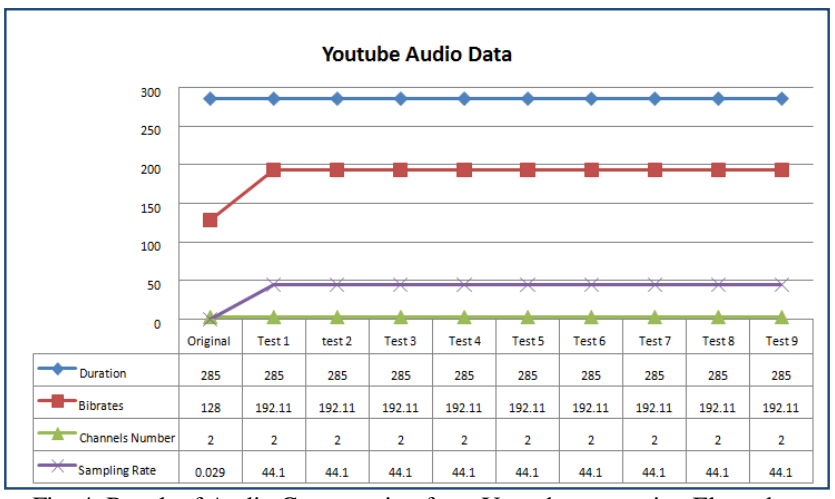

Fig. 4. Result of Audio Compression from Youtube.com using Elecard Video Format Analyzer

\section{CONCLUSIONS}

The analyst considered the video compression of social networks and has discovered both key similarities and contrasts between the compression rates through metadata extraction of the social networks as represented in the charts. With regards to the video and sound compression in Facebok.com, it is desirable to note that the compression is higher since video transferring in turns into an additional feature on the social network.

The metadata extraction result for Youtube.com, in any case, utilizing the video and audio compression parameters, is amazing as the webpage's fundamental element is to provide video spilling, transferring and downloading video information. With its structure and calculation, it is noted that Youtube.com gives minimal compression rate.

\section{ACKNOWLEDGMENT}

We would like to thank Causal Productions for permits to use and revise the template provided by Causal Productions. Original version of this template was provided by courtesy of Causal Productions (www.causalproductions.com).

\section{REFERENCES}

[1] Obar, et.al, (2015). Social media definition and the governance challenge: an introduction to the special issue". Telecommunications policy. 39 (9): 745-750.

[2] Davis, M. (2015). Facebook close sets speed record for $\$ 250$ billion market cap". Retrieved from: https://www.bloomberg.com/news/articles/2015-07-13/facebook-sclose-sets-speed-record-for-250-billion-market-value. Retrieved January 28, 2017

[3] Seymour, C. (2014). The growing impact of social media on video. Retrieved from: http://search.proquest.com/pqrl/docview/1671016132/E824440F5C9 24E6CPQ/1 ?accountid=36184. Retrieved on: February 1, 2017 
[4] Greenemeier, L., (2013). When will the internet reach its limit (and how do we stop that from happening)?. Retrieved from: https://www.scientificamerican.com/article/when-will-the-internetreach-its-limit/Retieved: February 6, 2017

[5] Rodriguez, et. Al., (2012). Quality metric to assess video streaming service over tcp considering temporal location of pauses (pdf). IEEE Transactions on Consumer Electronics. IEEE. pp. 985992. doi:10.1109/TCE.2012.6311346. Retrieved November 25, 2016

[6] Ross, S. (2015), Hashtags, algorithmic compression, and Henry James's late style. Retrieved from: http://search.proquest.com/pqrl/docview/1655115592/E0EA804D1A B94335PQ/2?accountid=36184 Retrieved on: February 1, 2017

[7] Poynton, C., (2012). Digital video and HD algorithms and interfaces. Morgan Kaufman Publishers of Elsevier, 225 Wyman Street, Waltam MA 02451 United State of America, page 147

[8] Zeng, M. (2004). "Metadata Types and Functions" NISO. Retrieved from: https://en.wikipedia.org/wiki/MetadataRetrieved on: February 1,2017

[9] National Information Standards Organization (NISO) (2001). Understanding Metadata (PDF). NISO Press. p. 1. ISBN 1880124-62-9.

[10] Webcase, Weblog (2011). "Examining video file metadata". Retrieved 25 November 2015
[11] Poynton, C., (2012). Digital video and HD algorithms and interfaces. Morgan Kaufman Publishers of Elsevier, 225 Wyman Street, Waltam MA 02451 United State of America, page 172-174.

[12] Facebook, (2017). What video file formats can I upload?. Retrieved from: https://www.facebook.com/help/218673814818907 Retrieved on: February 6, 2016

[13] Tinic Uro (2005). "The quest for a new video codec in Flash 8". Retrieved January 27, 2011. We went this route before with Sorenson Spark which is an incomplete implementation of H.263 and it bit us badly when trying to implement certain solutions.

[14] Adobe Systems Incorporated (2010). "Adobe Flash Video File Format Specification Version 10.1" (PDF). p. 72. Retrieved January 27, 2011. Sorenson $\mathrm{H}$

[15] "Market Demand for Sorenson Media's Sorenson Spark Video Decoder Expands Sharply". Sorenson Media. June 2, 2009. Retrieved July 31, 2009.

[16] Lowensohn, Josh (2010). "YouTube now supports 4k-resolution videos". CNET. Retrieved July 10, 2010.

[17] Schroeder, Stan (2015). "You can watch an 8K video on YouTube in theory". MashableUK. Retrieved July 2, 2015.

[18] Steven,Robertson (2016). "True colors: adding support for HDR videos on YouTube". Google. Retrieved January 17, 2017. 